\title{
UNUSUAL GALACTIC COSMIC RAY INTENSITY AND SPECTRAL CHANGES OBSERVED BY $V 1$ NEAR THE HELIOPAUSE
}

\author{
W. R. WebBer ${ }^{1}$ and J. J. Quenby ${ }^{2}$ \\ ${ }^{1}$ New Mexico State University, Astronomy Department, Las Cruces, NM, USA \\ 2 Blackett Laboratory, Imperial College, London, SW7 2BZ, UK \\ Received 2015 March 10; accepted 2015 May 3; published 2015 June 12
}

\begin{abstract}
We discuss here two unusual increases of cosmic ray intensity that were observed by $V 1$ in the last 1.1 AU before it crossed the heliopause in 2012 August, at 121.5 AU. These two increases are roughly similar in amplitude and result in a total increase in $\sim 1 \mathrm{GV}$ cosmic ray nuclei of over $50 \%$ and $0.01 \mathrm{GV}$ electrons of a factor $\sim 2$. During the first increase the changes in the $B$ field are small. After the first increase the $B$ field changes become large and during the second increase the $B$ field variations and cosmic ray changes are correlated to within \pm one day. During these time intervals, the rigidity dependence of the increases of GCR H and He nuclei from 100-600 MeV/nuc resemble those used to describe the solar modulation near the Earth during a large transient decrease but the ratio between the intensity changes of $\mathrm{H}, \mathrm{He}$, and electrons are different. The magnitude of these increases at Voyager is $\sim 1 / 3$ of the modulation that is required to produce the total modulation of protons, helium nuclei, and electrons between the local interstellar intensities and those observed at the Earth at the 2009 sunspot minima. This may imply that a significant part of the residual solar modulation at times of sunspot minima occurs near the heliopause itself.
\end{abstract}

Key words: cosmic rays - diffusion - interplanetary medium - magnetic fields - Sun: heliosphere

\section{INTRODUCTION}

Understanding the magnitude of the heliospheric modulation of the local interstellar cosmic ray flux is important for a variety of cosmological, astrophysical, and solar-related phenomena. Current interest in the possible dark matter annihilation origin of local positron and antiproton fluxes (e.g., Cirelli et al. 2008) requires correction for flux energy and intensity loss of the particles before they reach the Earth. Similarly, de-modulation of heavy nuclei (e.g., Lave et al. 2013) and proton fluxes (Adriani et al. 2013) seen locally is necessary in order to establish the local interstellar spectra relevant to studying comic ray origin. Investigation of long term variability in the production of cosmogenic radio nuclei (Beer et al. 2012) and in possible cosmic ray solar weather effects (e.g., Usoskin et al. 2011) depend on understanding the integrated effect of solar modulation from the Earth to local interstellar space. It is significant that all but one of the works just mentioned characterize this modulation employing the modulation potential approximation introduced by Gleeson \& Axford (1968). Adriani et al. (2013) is an example of the use of an integration of a 3D steady state model which improves accuracy at lower energies. It is the purpose of this paper to put forward new data from Voyager indicating that a significant fraction of the total heliospheric modulation occurs in a region close to the solar wind-local interstellar medium boundary where unexpected large intensity gradients are found when $V 1$ crossed the heliopause.

When $V 1$ crossed the heliopause on or about 2012 August 25th (day 238), there were extraordinary changes in the magnetic field and the energetic particle intensities (Burlaga et al. 2013; Stone et al. 2013; Webber \& McDonald 2013). On that date the particle intensities and field strength and direction began a change to values that have remained relatively unchanged now for over 20 months. Prior to this "final" event there were several unusual features in the energetic particle intensities that occurred that seem to have been lost in the excitement of the heliopause crossing. For energetic particles we mean GCR nuclei above 100 and 5-12 MeV electrons. The first of these intensity-time features occurred about May 7th (day 128) when $V 1$ was $1.1 \mathrm{AU}$ inside the heliopause when both the GCR nuclei and electrons increased by $\sim 15 \%$ and $20 \%$, respectively. After reaching these higher levels near the end of May (day 150), these intensities remained nearly constant for $\sim 58$ days ( $\sim 2$ solar rotation periods). Meanwhile the anomalous cosmic rays (ACR) intensities did the opposite, decreasing by $\sim 20 \%$ to a lower level where they also remained nearly constant for a 58 day time period.

On about July 28th (day 210), GCR nuclei and electron intensities increased suddenly for the second time. This second increase was more rapid and eventually larger ( 20\% and 40\%, respectively, for nuclei and electrons) than the first increase. However, the increase occurred in several stages, the final one starting on August 25th (day 238), the day of the heliopause crossing. At this time the GCR nuclei increased to their final values which were $\sim 32 \%$ higher than they were before May 7 th for $>70 \mathrm{MeV} /$ nuclei and $\sim 96 \%$ higher for $7-100 \mathrm{MeV}$ electrons. The "trapped" nuclei, termination shock particles (TSP), and ACR, disappeared suddenly (Krimigis et al. 2013), so that within just a few weeks the intensities of $2 \mathrm{MeV}$ protons were less than $0.1 \%$ of their intensity before May 7 th.

The magnetic field changes, both in amplitude and direction, are a crucial backdrop to these energetic particle changes.

These intensity changes of GCR and electrons are as large or larger than any transient decreases in this radiation seen at the Earth. At the Earth after a few days or weeks the intensity recovers to its pre-decrease level. At Voyager these increases are "steps" up to new and unprecedently high levels.

In this paper we will discuss the GCR and magnetic field temporal changes during this overall time period from day 128 to 238 when $V 1$ moves outward $\sim 1.1$ AU. We will then present the rigidity dependence of the observed intensity increases for 


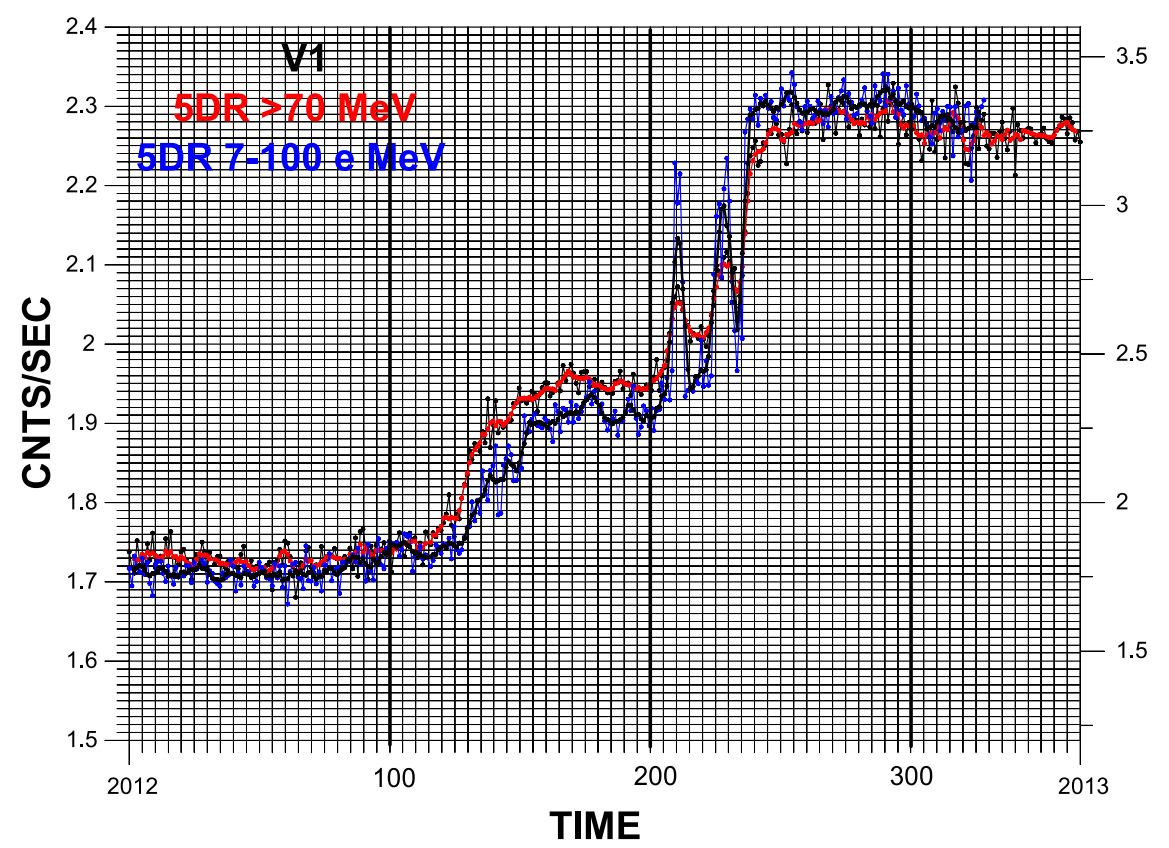

Figure 1. Five day running average of GCR $>70 \mathrm{MeV}$ (mostly nuclei) and 5-60 MeV (mostly GCR electrons). The two increases starting on day 128 and 208 are the solar modulation events discussed in this paper.

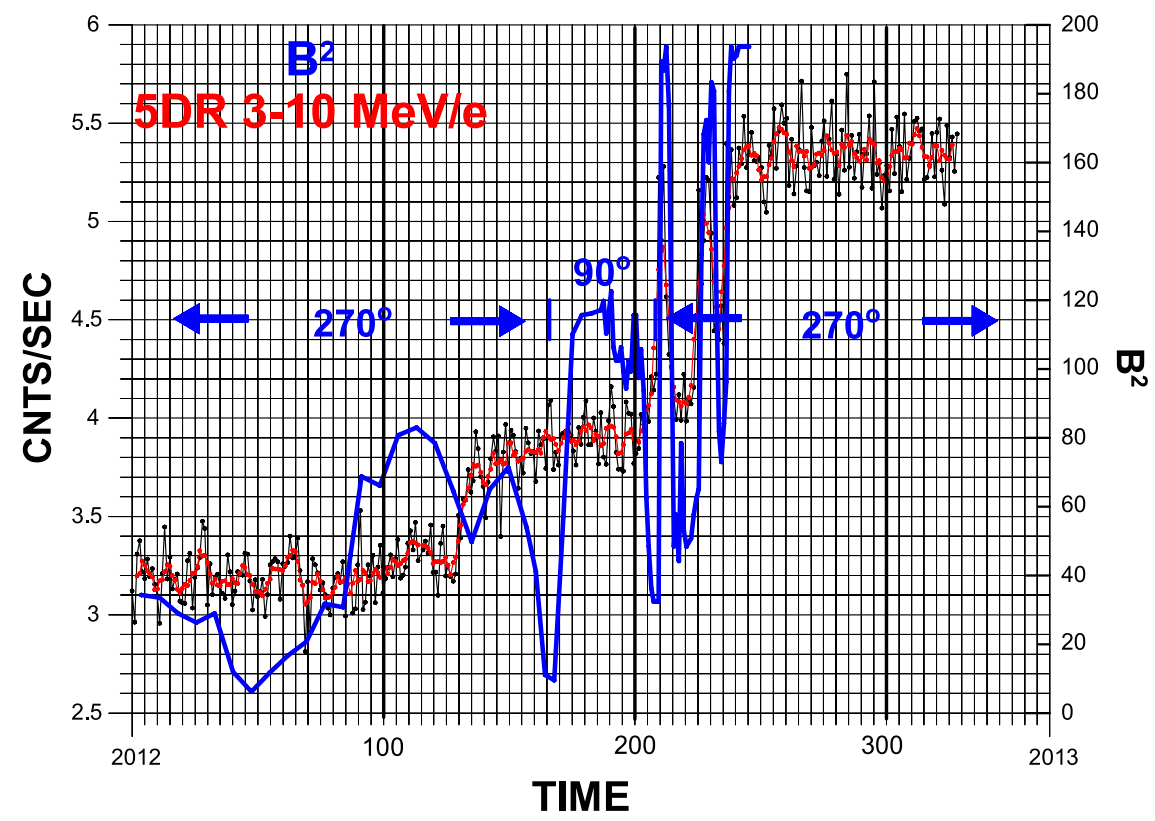

Figure 2. Similar to Figure 1 with the red line = five day running average except now for $3-10 \mathrm{MeV} /($ mostly GCR electrons), along with the relative energy density $\left(B^{2}\right)$ and direction of magnetic field $\left(90^{\circ}=\right.$ spiral field angle $)$.

the individual species $\mathrm{H}, \mathrm{He}$, and electrons to help define the mechanisms responsible for the intensity changes.

\section{THE INTENSITY-TIME CHANGES AND A DISCUSSION OF THEIR IMPLICATIONS}

The data presented here from $V 1$ clearly show two periods of increase for both GCR nuclei and electrons. This is illustrated in Figure 1 which shows the integral rates of $>70 \mathrm{MeV}$ nuclei and 5-60 MeV electrons. The total increase for each component from before May 7th to after August 25th (32\% for $>70 \mathrm{MeV}$ and $96 \%$ for $5-60 \mathrm{MeV}$ electrons) are made equal in the plot using different scales on the left hand and right hand axis. This is to show the relative magnitude of the increase on May 8th to that in the second change between July 28th and August 25th for the two species. The intensity changes in the two increases are not identical in magnitude or in their relationship to the magnetic field.

The format in Figure 2 is similar to Figure 1 and shows the 3-10 MeV electrons (left hand scale) and the relative magnetic energy density $\sim B^{2}$ (right hand scale), as derived from plots of the $B$ field amplitude presented by Burlaga $\&$ Ness (2014). For the $3-10 \mathrm{MeV}$ electrons the total increase is $69 \%$, which is less than the increase of $96 \%$ for the $5-60 \mathrm{MeV}$ electrons.

For the $B$ field, shown in Figure 2, the changes in amplitude are over a factor of 4 during this time period from values $\sim 1$ to 
$\sim 4.5 \mu \mathrm{G}$ (the final field value) in just a few days from days 208 to 210 . There are two changes in $B$ field direction (days 163-172) from a positive to a negative polarity and a much more sudden and final change on days 208-209, from negative to positive polarity. These field changes are related to specific energetic GCR intensity charges in this paper. These changes in the $B$ field and the integral cosmic ray intensity are noted by Burlaga \& Ness (2014).

We note here that the first step in the cosmic ray intensity changes at day 128 does not occur in association with any major $B$ field amplitude change. After this GCR intensity change and later during the period of constant GCR intensity, the $B$ amplitude decreases and then increases, however, by a factor of 3 between days 162 and 172, during a 10 day period when the field direction is also changing from $270^{\circ}$ to $90^{\circ}$. During this 10 day period the field inclination increases from $\sim 0^{\circ}$ to $\sim 90^{\circ}$ (Burlaga et al. 2013). The locally measured GCR intensities for all of these charges were unresponsive to these specific extraordinary $B$ field changes!

The next field polarity change occurred on day 209 and is the final decisive polarity change from $90^{\circ}$ to $\sim 270^{\circ}$. One day later the $B$ field amplitude changes by a factor 3 in one day to its final value of $4.5 \mu \mathrm{G}$ (Burlaga \& Ness 2014). These $B$ field changes that occur between days 210 and 238 are matched within \pm 1 day by corresponding changes in GCR nuclei and electrons and also at the same time by opposite changes in TSP and ACR as seen in Figure 2 (see also Krimigis et al. 2013 and Burlaga et al. 2013). Also, as seen in Figure 2, the lower rigidity electrons are more responsive than the GCR nuclei to these changes in field amplitude that pass $V l$ between days 210 and 238.

So overall we have the observation by $V 1$ that the first GCR increase starting on day 128 was not coincident with the correspondingly large $B$ field amplitude or direction changes. A following period of nearly constant GCR intensity, however, was coincident with large amplitude and direction changes of the $B$ field, as well as unusual $B$ field elevation angle changes.

And the second GCR increase starting on day 208 and the following intensity changes culminating with the final increase of GCR on day 238, were all simultaneous within \pm 1 day with the very large $B$ field magnitude changes, but there were no $B$ field direction changes during this time. The lower rigidity electrons were again more responsive to these $B$ field changes than the GCR nuclei whose rigidity is $\sim 50$ times that of the electrons. In this second increase of GCR, the TSP and ACR intensity changes were again opposite to the GCR to within \pm 1 day as these components disappeared.

\section{SPECTRAL CHANGES OF GCR PROTONS, HE NUCLEI, AND ELECTRONS BETWEEN DAY 128 AND 238 OF 2012}

In the Stone et al. (2013) article, in Figures 2-4 the intensities and spectra of $\mathrm{H}$ and $\mathrm{He}$ nuclei and electrons are shown for time periods before May 8th and after August 25th. This includes the period of the two GCR increases. Below an energy of $\sim 80 \mathrm{MeV} / \mathrm{nuc}$, the time period before May 8 th is contaminated by background ACR intensities for $\mathrm{H}$ and $\mathrm{He}$ nuclei and therefore these energies cannot be used in the comparison, so for $\mathrm{H}$ and $\mathrm{He}$ nuclei the energy range covered by the data is $\sim 100-600 \mathrm{MeV} /$ nuc and for electrons $\sim 10-40 \mathrm{MeV}$. In these energy ranges a comparison of the intensities before and after the heliopause crossing can be made using the data in Stone et al. (2013) and also Webber \& McDonald (2013).

The intensity changes of these particles with different mass to charge ratio, $A / Z$ have, historically been very useful for understanding the origin of solar modulation effects. For example, Gleeson \& Axford (1968) have compared the H and He intensity changes in their study of the solar modulation. These relative intensity changes also appear in the FokkerPlanck formalism which we use (e.g., Fisk \& Axford 1969). It is found that if the changes in intensity, $\beta \ell n\left(j_{1}(P) / j_{2}(P)\right)$, are plotted as a function of rigidity there is a splitting of the modulation for each species according to their charge to mass ratio $Z / A$. This splitting arises from the fact that the modulation itself, expressed in $\mathrm{MeV}$, is defined by a modulation function given by the expression $\Psi=\mathrm{Ze} \int(V / 3) d r / K(r)$, where the diffusion coefficient $D=\beta K(r) P$ is a scalar coefficient, which has a $P$ dependence $\sim \beta P$ and $V$ is the radial solar wind speed. Gleeson \& Axford (1968) also introduced another quantity called the modulation potential $\phi=\int(V / 3) d r / K(r)$ expressed in MV. This modulation potential is the same for $\mathrm{H}, \mathrm{He}$ and electrons at the same rigidity. The modulation function, $M$, that is commonly used, is defined by $\Psi$. $M$ describes the amount of modulation at two different times (or places) and is different at the same rigidity for particles of different $A / Z$, hence the use of the term charge splitting when this quantity is used (see the section by Ken McCracken, pp. 50-58, in the book Cosmic Radio Nuclei by Beer et al. 2012 for a discussion of the two quantities, modulation function and modulation potential). The modulation function is useful for comparing intensity changes of the different species and is widely used (see the above book for an extensive list of references on the application of this model).

Figure 3 is a plot of $\beta \ell n\left(j_{2} / j_{1}\right)$ versus $P$ for Voyager data on $\mathrm{H}$ and $\mathrm{He}$ nuclei before and after the heliopause crossing as compared with a solar modulation potential of $80 \mathrm{MV}$ acting on the interstellar spectrum. Note the charge splitting of the amount of solar modulation in the calculation which results in a two times greater modulation for $\mathrm{H}$ than $\mathrm{He}$ at rigidities $\leqslant 1 \mathrm{GV}$. The data points are for the observed modulation at Voyager 1 of $\mathrm{H}$ and $\mathrm{He}$ from before May 8th to after August 25 th. The intensity changes seen at Voyager 1 are roughly consistent with modulation model calculations, which use the Fokker-Planck equation as originally supplied to us many years ago by H. Moraal (2004, private communications). An overall modulation of $\sim 80 \mathrm{MV}$ is needed to fit the data but with no obvious charge splitting seen in the data.

The observed modulation function for electrons at Voyager at lower rigidities is independent of $P$ and is $\sim 2$ times that for $\mathrm{H}$ and He nuclei at $\sim 0.5 \mathrm{GV}$ which is in agreement with the assumption that the diffusion coefficient is independent of $P$ below $1 \mathrm{GV}$. This is consistent with the assumption used by Potgieter \& Nndanganeni (2013) to derive the interstellar electron spectrum from Voyager measurements of electrons in the heliosheath.

\section{GENERAL COMMENTS}

It is not the intent of this paper to develop a theoretical model for an explanation of these modulation effects observed in the outer heliosheath at $V I$ by the CRS instrument. We believe that the GCR intensity changes are so unusual and unprecedented in the history of cosmic ray studies that they are not easily accommodated within the Parker (1963) heliosphere 


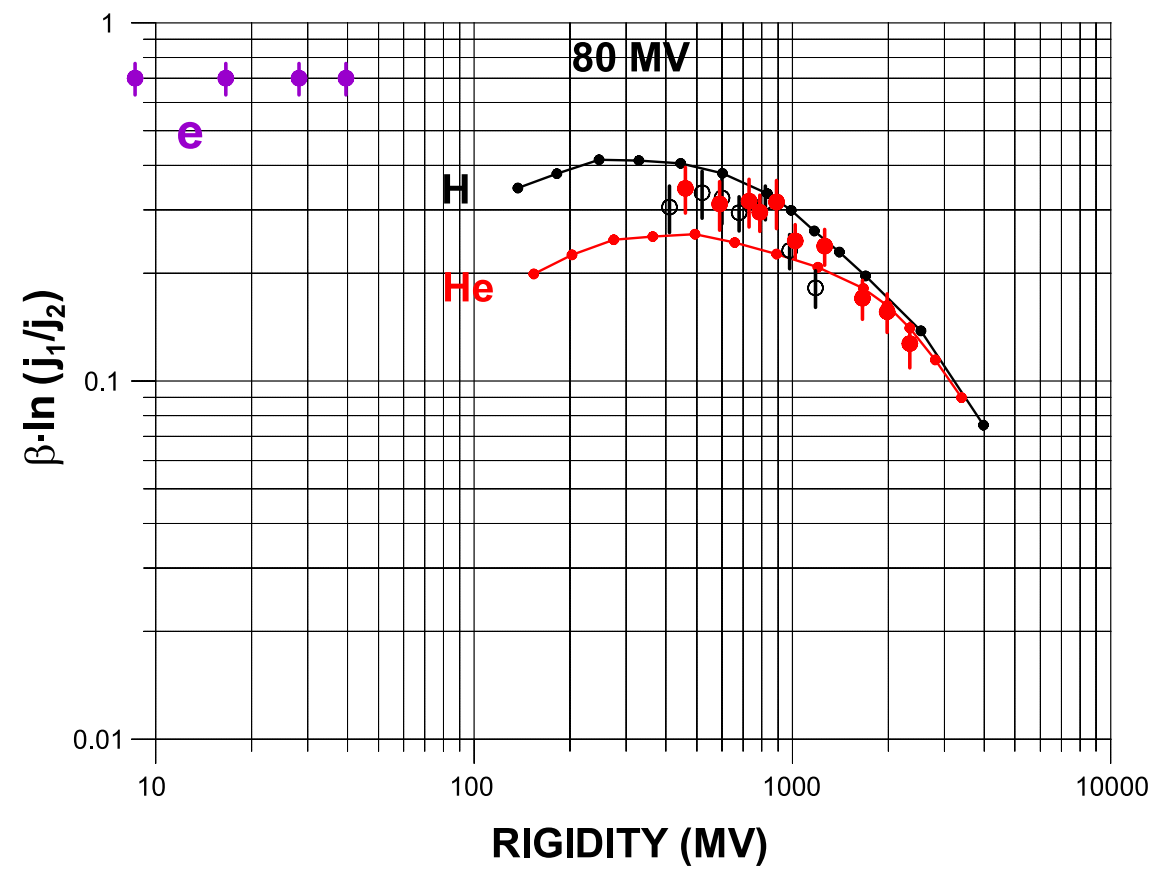

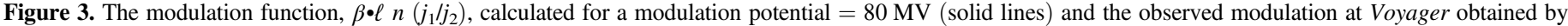
comparing $\mathrm{H}, \mathrm{He}$, and electron spectral intensities measured before day 128 and after day 238 of 2012 . The details are discussed in the text.

modulation picture as developed by many others, e.g., Gleeson \& Axford (1968) and Fisk \& Axford (1969), including a simple force field solution. The overall modulation used to describe the cosmic ray changes at the Earth is still based on these earlier concepts and is used widely in many disciplines (e.g., Beer et al. 2012).

But there are certain features of the observed modulation near the heliopause that may indicate that the characteristics of the $B$ field, plasma flows, etc., affect the entrance of the LIS cosmic rays into the heliosphere. And these "heliopause" effects may play a significant part in the overall heliospheric modulation inside the heliopause. Many articles are now appearing on these effects and we recognize that of Guo \& Florinski (2014).

The first step in this modulation process near the heliopause is related to the first event that started about May 8th (DOY 128). This event contributed about $40 \%$ of the total increase for both GCR nuclei at higher rigidities and electrons at lower rigidities. The changes in the $B$ field were small during the 10-20 day time period of the first increase as noted earlier. During the following $\sim 58$ day time period up to about day 200 the intensity of the GCR remained relatively constant at the higher intensity level to within a few percent. However, during this time period the $B$ field recorded some of the largest changes yet seen at $V 1$ with a polarity change from $270^{\circ} \rightarrow 90^{\circ}$, along with unprecedented changes in the elevation angle near the end of this constant GCR intensity period.

The lack of significant time correlation between GCR intensity changes and $B$ field changes during the time period from DOY 128-208 suggests that the GCR changes during the first increase could be related to much larger scale features that may not be evident in the local field being measured at $V I$ at that time.

This lack of correlation between B and GCR intensities in the first event is definitely not present in the second event which started on July 28th (day 208). In this event, from July 28th to August 25th, the GCR and $B$ field changes were correlated to within \pm 1 day or less. This correlation continues through all five intensity increases and decreases, all of them exceptional, until the final increase on August 28th (day 238). In each of these increases the $B$ field magnitude and direction at the times of the $B$ field maxima was essentially the same as that observed after August 28th. The GCR electrons and nuclei, however, did not reach the maximum intensities that were observed after August 28th. For electrons from 5-60 MeV the peak increases were $\sim 80 \%$ of the post-August 28 th intensity. For nuclei, the increases reached levels $\sim 50 \%-60 \%$ of the post August 28th intensity, so there is a distinct rigidity dependence of the GCR distribution within these structures that pass $V 1$. Note that the outward speed of $V 1$ is $\sim 0.01 \mathrm{AUday}^{-1}$, so the \pm 1 day correlation between $B$ and the GCR that has been observed could have a scale $\sim 0.01$ AU (e.g., Stone et al. 2013)!

The intensity changes of electrons and $\mathrm{H}$ and $\mathrm{He}$ nuclei as a function of rigidity for the overall time period from day 128 to 238 covered in this study are large and well defined. At least two features of the $\beta \cdot \ell n\left(j_{1} / j_{2}\right)$ versus $P$ data for this modulation of the different species shown in Figure 3 are important: (1) the observed intensity changes of electrons are nearly independent of rigidity at low rigidities. In addition, if the values of electron modulation function $\beta \cdot \ell n\left(j_{\mathrm{LIS}} / j_{2}\right)$ at low rigidities are extrapolated to higher rigidities, it has a value $\sim 2$ times the value of the modulation function observed for $\mathrm{H}$ and $\mathrm{He}$ nuclei at about $0.5 \mathrm{GV}$. (2) The $\mathrm{H}$ and He nuclei have observed values of the modulation function that are similar to each other. The splitting in the modulation of these charges has been observed in the normal inner heliospheric modulation of these different particles (e.g., Lezniak \& Webber 1971).

In the spherical modulation model we have used, the average magnitude of the modulation function for $\mathrm{H}$ and $\mathrm{He}$ as a function of rigidity for this final time interval would be 
equivalent to that for a modulation potential $=80 \mathrm{MV}$ as indicated in Figure 3.

In fact, in this same model, starting with estimated LIS spectra, a modulation potential $=250 \mathrm{MV}$ has been used to reproduce the carbon and heavier nuclei spectra measured by $A C E$ in 2009-2010 at the Earth, (e.g., Lave et al. 2013) as well as the spectrum of $\mathrm{H}$ nuclei observed by PAMELA in 2009 (Webber et al. 2014). Thus the modulation observed by Voyager 1 in the last $1.1 \mathrm{AU}$ of the heliosheath could be an important part of to the overall solar modulation observed at the Earth, contributing possibly as much as $\sim 1 / 3$ of the overall modulation potential observed at the Earth at this time of the solar cycle. A possible model to explain some of the new modulation features is described in Quenby \& Webber (2014).

\section{SUMMARY AND CONCLUSIONS}

This paper describes two large and unprecedented modulation events of GCR observed at VI starting on May 8th and July 28th just prior to the crossing of the heliopause on 2012 August 28 at a distance of 121.7 AU. These events resulted in the increase of GCR electrons from $5-60 \mathrm{MeV}$ by a factor $\sim 2$ and $\mathrm{H}, \mathrm{He}$, and nuclei above $\sim 0.5 \mathrm{GeV}$ to increase by factors of up to 3 at the lowest rigidities. Although these increases are complex, the overall intensity changes as a function of rigidity for protons, helium nuclei, and electrons that are observed by Voyager can be represented by a modulation potential change of $\sim 80 \mathrm{MV}$ which would amount to $\sim 1 / 3$ of the total modulation potential required to reproduce the spectra of these same nuclei observed at the Earth at a time of sunspot minimum in 2009 (e.g., Mewaldt et al. 2010). Thus a new and significant feature is added to the description of the already unusual solar modulation of GCR in the heliosheath (e.g., Potgieter et al. 2013).

The first modulation event occurred when $V 1$ was $1.1 \mathrm{AU}$ inside the HP. The intensity increases starting on May 8th (day 128) and continuing up to day 150 amounted to about $40 \%$ of the combined increase of both events. During this GCR increase there were only modest changes, both positive and negative, in the $B$ field amplitude with no changes in the direction. For the next 60 days the GCR and ACR intensities remained almost constant; however, between days 150 and 160 the $B$ field first changed direction from $270^{\circ}$ to $90^{\circ}$ and then decreased by a factor $\sim 2.0$, followed in a few days with a sudden increase by a factor $\sim 3$ accompanied by an increase in elevation angle from $\sim 0^{\circ}$ to $90^{\circ}$. It almost seems like the $B$ field was turning itself inside out over a period of a few days, but without any observable effects on GCR or ACR.

The $90^{\circ}$ polarity time period ended suddenly on July 28th (day 208) when the polarity changed to $270^{\circ}$ followed by an increase in the magnitude of the $B$ field (on day 209) again by a factor $\sim 3$ to essentially its final value $\sim 4.5 \mathrm{uG}$ after day 238 .

This increase on July 28th and the subsequent changes in B were coincident within 1 day with corresponding positive and negative intensity changes of GCR. In this period the changes in ACR (Stone et al. 2013) and TSP (Krimigis et al. 2013), were exactly opposite to those of GCR. The details of these changes provide a glimpse into features of the heliopause with structures possibly of a scale $\sim 0.01 \mathrm{AU}$. And these structures could be moving with speeds much greater than $V 1$.

This second GCR modulation increase was embedded in massive $B$ field fluctuations in contrast to the first modulation increase in which these $B$ field changes were small. In the first increase the $B$ field magnitude and polarity changes actually occurred after the GCR increase and when the GCR changes themselves were small. The first and second GCR increases have roughly the same magnitude and same rigidity dependence, however, despite their greatly different correlation with the $B$ field. They could be part of a larger structure $\sim 1 \mathrm{AU}$ in extent that characterizes the heliopause region.

An over-riding feature of this data is the complexity of the intensity changes of both the $B$ field and GCR nuclei and electrons on scales as small as 0.01 AU. The correlation and lack of correlation of these changes is evident in the second and first modulation events, respectively.

In an astrophysical sense this heliopause, which may be present in millions of cases in the galaxy where there are stellar winds on all kinds of scales, is notable for the energy it removes from local GCR rather than the acceleration of any particular particle population.

W.R.W. wishes to thank his Voyager colleagues from the CRS instrument, Project PI Ed Stone, Alan Cummings, Nand Lal, Bryant Heikkila, and the late Frank McDonald. Support from JPL is greatly appreciated.

\section{REFERENCES}

Adriani, O., Barbarino, G. C., \& Bazilevskaya, G. A. 2013, ApJ, 765, 91 Beer, J., McCracken, K., \& von Steiger, R. 2012, Cosmic Radio Nuclei, (Berlin: Springer-Verlag)

Burlaga, L. F., Ness, N. F., \& Stone, E. C. 2013, Sci, 340, 150

Burlaga, L. F., \& Ness, N. F. 2014, ApJ, 784, 146

Cirelli, M., Franceshini, R., \& Strumia, A. 2008, NuPhB, 800, 204

Fisk, L. A., \& Axford, W. I. 1969, JGR, 74, 4973

Gleeson, L. J., \& Axford, W. I. 1968, ApJ, 154, 1012

Guo, X., \& Florinski, V. 2014, ApJ, 793, 18

Krimigis, S. M., Decker, R. B., \& Roelof, E. C. 2013, Sci, 344, 144

Lave, K. A., Wiedenbeck, M. E., \& Binns, W. R. 2013, ApJ, 770, 117

Lezniak, J. A., \& Webber, W. R. 1971, JGR, 77, 1805

Mewaldt, R. A., Davis, A. J., \& Lave, K. A. 2010, ApJ, 723, 1

Parker, E. N. 1963, Interplanetary Dynamical Processes (New York: Interscience Publishers)

Potgieter, M. S., \& Nndanganeni, R. R. 2013, APh, 48, 25

Quenby, J. J., \& Webber, W. R. 2014, arXiv:1409.1105

Stone, E. C., Cummings, A. C., \& McDonald, F. B. 2013, Sci, 341, 150

Usoskin, I., Bazileskya, G., \& Kovaltsov, G. A. 2011, Proc. 32nd ICRC (Beijing), 11, 39

Webber, W. R., \& McDonald, F. B. 2013, GeoRL, 40, 1665 